\title{
Examining the Influence of Social Media Education Through Mobile- Phone on the Change in Physical Activity Behavior of Pregnant Women: An Intervention Study
}

\section{Erfaneh Talebi}

Urmia University of Medical Sciences

Hamideh Mohaddesi

Urmia University of Medical Sciences

Davoud Vahabzadeh ( $\sim$ dvaha2003@yahoo.com )

Ilam University of Medical Sciences

Javad Rasuli

Urmia University of Medical Sciences

\section{Research Article}

Keywords: Education, Social Network, Behavior Change, Physical Activity, Pregnancy

Posted Date: August 30th, 2021

DOl: https://doi.org/10.21203/rs.3.rs-714947/v1

License: (c) (i) This work is licensed under a Creative Commons Attribution 4.0 International License.

Read Full License 


\section{Abstract}

Background: Nowadays, a new model of health approach through mobile-phones is widely used to deal with improving physical activity and its beneficial effect on pregnancy. This study was aimed to determine the influence of social networking through mobile phones on changing the physical activity behavior in pregnant women.

Methods: This randomized interventional study was conducted with parallel groups on 90 pregnant women referring to Urmia Health centers in 2018-2019. The participants from various social and economic backgrounds were randomly included. The participants were randomly assigned to a control and a treatment group. Demographic and Pregnancy Physical Activity Questionnaire (PPAQ) questionnaires were filled. In addition to routine care, the treatment group received educational intervention through social network within 16 training sessions related to physical activities and nutrition in 8 weeks.

Results: The mean physical activity level and weight in both control and treatment groups differed significantly after intervention $(P<0.001)$. At the beginning of the study, control and treatment groups were not different in terms of daily physical activity level $(P=0.17)$, meanwhile, there was a statistically significant difference at the end of intervention in the level of daily physical activity $(P<0.001)$. Comparison of both groups, based on different categories of activity level at the end of the treatment, showed a non-significant difference in sedentary activities $(P=0.89)$, but the intervention led to a significant difference based on the other level of activities; light $(P<0.001)$, medium $(P<0.001)$, and intense $(P=0.05)$.

Conclusion: Educational intervention based on social networks through mobile phones had an effective role in behavior change in physical activity during pregnancy.

Trial registration code: IRCT20151004024340N15, Registration date: 2020/06/05

\section{Introduction}

By the change in social and economic patterns all over the world, the sedentary lifestyle has turned into a global phenomenon [1]. A sedentary lifestyle is accompanied by overweight, type 2 diabetes, and cardiovascular diseases; therefore, improving lifestyles is one of the paramount important action for public health [2]. Pregnancy is considered the most sensitive phase in a mother's life. Having a healthy lifestyle during this period is very important as the fetus is so sensitive and vulnerable to various factors at this time, and the mother's health has a direct influence on fetal life [3].

According to documents and scientific research, there is a positive relationship between physical activity and the health of pregnant mothers and their children [4]. Physical activity, which includes playing, walking, doing house chores, gardening, dancing, etc., is introduced as any kind of body movement that is performed through skeletal muscles. Although such activities consume energy, they should not be 
confused with sports [5]. American College of Obstetrics and Gynecology suggests physical activity during pregnancy and US instructions recommend healthy pregnant women to practice more than 30 min of physical activity on most days of the week, if there are no any raised side effects for the fetus [6]. Physical activity is an effective way to reduce unpleasant influences of pregnancy such as sleeplessness and feeling tired [7], excessive weight gains of a mother [8], back and pelvic pain [9], constipation [10], high blood pressure [11], depression and stress [12]. Such practices can reduce the severity of pain during labor, improve heart and lung functions, and in the post-labor phase, helps the body return to normal quickly [13]. Despite such advantages, the studies demonstrate a significant reduction in the physical activity of women during pregnancy $[14,15]$. In this regard, it has been shown that only $23 \%$ of pregnant women in America performed the minimum physical activity suggested by the American Congress of Pregnancy and Gynecology $[16,17]$. Studies have identified some relevant factors with a reduction in physical activity levels in pregnancy, including the lack of training by health care staff about the benefits of physical activity or recommendations to perform safe physical activity in pregnancy [10].

Since the invention of the World Wide Web in 1989, access to the internet and its use has been expanded. Connected devices such as cell phones and computers and kitchen appliances with internet connectivity have covered most aspects of our lives. Nowadays, people can connect and transfer information without any geographical or time barrier. The common term to describe such information and idea-sharing on the internet or mobile platforms is "social media", which is defined by the Oxford dictionary as "websites and programs that enable users to create and share content or to participate in social networks" [18].

Mobile phones are among the important appliances to use social networks, and their extensive access has made them a valuable option for interventions with the purpose of change in behavior $[7,19]$. Besides, increasing the availability of health information in a digital format (which can be easily observed and used) and allowing the quick direct interaction between the patient and caregiver are other benefits [20]. During the last decade, in countries with medium income, there has been an increasing research study on mobile health (mHealth). This concept is defined as the use of appliances such as mobile phones or smartphones to transfer health content and various services [5].

It has been reported that the mobile phone is the fastest technology utilized in low and high-income countries. Therefore, a new health approach is increasingly utilized nowadays through mobile phones. As a result, attempts to increase the physical activity levels in all populations can be put into practice by applying advanced programs of communication technology [21]. Research by Rothert et al. (2006) indicated that weight loss in participants was significantly higher in the group that used the modern technology system compared to the group who just received routine care [22]. Currently, the value of a training program depends on its impact on change or the creation of relevant behavior. As mentioned before, physical activities play a key role in improving pregnant women's health in society, reduction of obesity, pregnancy over-weight, and unbalanced pregnancy weight gain. Moreover, long-term and shortterm physical activities have benefits on health during pregnancy and after labor. Regarding these issues, as well as the influence of maternal health on future generation health and the ease of access to the 
internet and its pervasiveness, the current study was designed to determine the effect of social networkbased training via mobile phones for changing physical activity pattern of pregnant women.

\section{Methods}

\section{Design and sampling:}

This randomized interventional study with parallel groups was conducted on pregnant women referred to Urmia health centers in 2018-2019. The study was registered by the ethics code of IR.UMSU.REC.1397.162 in Ethics Committee of Urmia Medical Sciences University and by IRCT20151004024340N15 in International Center for Clinical Trials Registrations.

\section{Sample size and sampling:}

The sample size was calculated according to Michele Bisson (Bisson et al., 2015) study, where the MVPA index was $11.7 \pm 9.5$ in the control group and $25.4 \pm 20.4$ in the treatment group after the intervention. Using the sample size equation for comparing the mean of two groups, $90 \%$ power $\left(Z_{1-\beta}=1.28\right)$, the twosided alpha level at $0.05\left(Z_{1-\alpha / 2}=2.575\right)$, and a $10 \%$ chance of dropout, the sample size was 45 subjects for each group. Therefore, ninety subjects were included in the study.

Inclusion criteria in the study were: the tendency to participate in the study, having an ability for reading and writing, under 20 weeks' gestational age, lack of chronic diseases (i.e., diabetes, hypertension, kidney disease), lack of any medical limitation for improving physical activity by mother, no mental illness and no history of hospitalization (according to participants' self-report), lack of cerclage and prenatal care, having a smartphone, access to the internet, and residence in Urmia. The exclusion criteria were: having any problems or special diseases in current pregnancy such as preeclampsia, diabetes, anemia, high-risk pregnancy and cerclage surgery, frequent bleeding during the intervention, premature contractions, reduction of fetal movements, amniotic fluid leakage, diet for a specific disease, and migration from Urmia.

At first, for sampling the health care centers were categorized into three levels; 1,2 , and 3 according to their social and economic situations. Then, two centers from each level were chosen randomly by lottery (drawing). Participants in the study were randomly assigned to the control and treatment group and the block randomization method was used for this purpose. First, the blocks were created with the combination of AAABBB by computer and all the possible statuses were identified and an exclusive code was assigned for each. Next, considering the sample size $(N=90)$ and block sizes $(S=6), 15$ blocks were chosen by a simple randomization method by the researcher. Selected subjects were allocated to control or intervention group consecutively by the study manager. All these stages were done under the provision of a consultant epidemiologist and using Random Allocation Software version 1.0.0.

\section{Data collection tools:}


In this study, demographic, Pregnancy Physical Activity Questionnaire (PPAQ) questionnaires recruited. Height measured with a Stadiometer and weight by a Seca scale

Demographic information questionnaire had questions such as age, weight, height, education level, employment status, economic status, BMI, pregnancy age, number of fetuses, number of pregnancies, history of the underlying disease, history of infertility, cerclage, ectopic pregnancy, and specific disease in the current pregnancy.

Standard PPAQ, which is related to physical activity in pregnancy, includes two parts: Part I consisted of information about individual characteristics and Part II includes 32 questions on physical activities. The questionnaire of pregnancy physical activity consists of 4 groups of questions related to activities: at home (16 questions), community (3 questions), and activity in the workplace (5 questions), and sports and entertainment (8 questions).

After selection of subjects in the first meeting, the researcher introduced herself to mothers and explained the study purpose. Informed written consent was then obtained. Later the questionnaires related to personal information and physical activity were filled in. Body mass index (BMI) was calculated and assessed. The mother's weight was checked with minimum clothing and without shoes, using a digital scale with a precision of $100 \mathrm{~g}$. People's height was measured using a wall gauge with an accuracy of 1 $\mathrm{cm}$ while standing barefoot by the wall, as the shoulders were in a normal position. BMI was calculated by dividing weight by height squared.

Based on weight status (i.e., normal, overweight, and obese) and through the permuted block randomization, all the pregnant women were randomly assigned to the control and treatment group by Random Allocation Software version 1.0.0. Both groups received individual diets according to BMI, by a nutritionist, who also provided the necessary explanations.

\section{Intervention content:}

The control group received a routine pregnancy care in health centers; subjects joined a virtual group in a social network, and received materials about their nutrition program and tracking its effect on their weight gain during pregnancy. In parallel, the treatment group also joined a virtual training group in a social network. They received educational content to improve their physical activity plus an individual diet during 16 sessions in 8 weeks. The content delivered to the virtual group twice a week and at a specified time. All women's questions were answered both in group and in private based on the type of question. To ensure the proper implementation delivered program by mothers, mothers' weights were recorded after a face-to-face meeting in the fourth week. All delivered massages deposited in virtual group by the end of intervention.

At the end of the intervention, pregnant mothers in both groups filled in the PPAQ questionnaires for the second time. The data acquired from current questionnaire were compared with the first one (before the treatment). 


\section{Data Analysis:}

Quantitative data were reported as Mean \pm Standard deviation and qualitative data were expressed in the form of frequencies and percentages. Activity intensity was measured based the Metabolic Equivalent of Task (MET). To calculate the total MET of activity, the MET level regarding the intensity of activities was multiplied by the amount of time spent per day. The level of daily activity can be determined based on the amount of MET obtained for activities and their average per day. An activity with MET less than 1.5 was considered as inactivity, with MET 1.5-3 as light activity, with MET 3-6 as moderate activity, and with MET more than 6 as an intense activity. An independent t-test was used to compare groups. A paired t-test was used for comparisons within groups (before and after). Also, the chi-square test was used to compare qualitative variables between the two groups. To control the possible influence of confounding factors and better determination of treatment effect, multivariate analysis was done. The significance level for all statistical tests was considered less than 0.05. All analyses were performed using SPSS-21 software.

\section{Results}

In this research, 45 subjects were assigned to the each group (Control and intervention group); however, finally 36 subjects in the control group and 35 subjects in the intervention group were succeeded in completing the study period. Two groups were exactly identical in terms of all basic properties. The quantitative and qualitative demographic characteristics of the participants in each group are shown in Table 1.

Table 1-Demographic characteristics of the intervention and control subjects 


\begin{tabular}{|c|c|c|c|c|}
\hline Variable & Sub Categories & Control & Intervention & \multirow[t]{2}{*}{$P$ value } \\
\hline & & Mean $\pm S D$ & Mean $\pm S D$ & \\
\hline Age & All & $28.94 \pm 5.33$ & $29.46 \pm 5.78$ & 0.7 \\
\hline Husband age & All & $33.58 \pm 5.38$ & $35.34 \pm 6.37$ & 0.21 \\
\hline Gravida & All & $2.00 \pm 1.15$ & $1.86 \pm 0.85$ & 0.55 \\
\hline Marriage duration & All & $6.30 \pm 4.53$ & $7.16 \pm 5.99$ & 0.50 \\
\hline Gestational Age & All & $12.03 \pm 5.34$ & $13.97 \pm 4.38$ & 0.10 \\
\hline BMI & All & $27.02 \pm 4.66$ & $27.17 \pm 4.37$ & 0.89 \\
\hline \multirow[t]{4}{*}{ Volunteers education level ( $\mathrm{N} \%)$} & Elementary & $2(5.6 \%)$ & $3(8.6 \%)$ & \multirow{4}{*}{0.63} \\
\hline & Middle school & $5(13.9 \%)$ & $5(14.3 \%)$ & \\
\hline & High school & $12(33.3 \%)$ & $7(20 \%)$ & \\
\hline & Collegiate & $17(47.2 \%)$ & $20(57.1 \%)$ & \\
\hline \multirow[t]{4}{*}{ Husband education level ( $\mathrm{N} \%)$} & Elementary & $3(8.3 \%)$ & $4(11.4 \%)$ & \multirow{4}{*}{0.79} \\
\hline & Middle school & $5(13.9 \%)$ & $5(14.3 \%)$ & \\
\hline & High school & $12(33.3 \%)$ & $8(22.9 \%)$ & \\
\hline & Collegiate & $16(44.5 \%)$ & $18(51.4 \%)$ & \\
\hline \multirow[t]{2}{*}{$\operatorname{Job}(\mathrm{N} \%)$} & Employed & $7(19.4 \%)$ & $13(37.1 \%)$ & \multirow[t]{2}{*}{0.1} \\
\hline & Unemployed & $29(80.6 \%)$ & $22(62.9 \%)$ & \\
\hline \multirow[t]{3}{*}{ Economic status ( $\mathrm{N} \%)$} & $>$ Expense & $6(16.7 \%)$ & $5(14.3 \%)$ & \multirow{3}{*}{0.96} \\
\hline & Equal to Expense & $22(61.1 \%)$ & $22(62.9 \%)$ & \\
\hline & $<$ Expense & $8(22.2 \%)$ & $8(22.8 \%)$ & \\
\hline
\end{tabular}

As shown in Table 2, the mean level of physical activity and weight in both groups after the intervention was significantly different from the start phase $(P<0.001)$. At the beginning of the study, the control and intervention groups did not differ from each other in terms of the physical activity level $(P=0.17)$, while there was a significant difference based on the level of activity at the end of the intervention $(P<0.001)$. Also, significant weight changes were seen in both groups in the post-intervention phase compared with the initial phase of the study $(\mathrm{P}<0.001)$, but the comparison of subjects based on the weight after the intervention did not show any significant difference between the two groups $(P=0.20)$.

Table 2 - Comparison of mean and standard deviation of physical activity and weight before and after the intervention in the two groups 


\begin{tabular}{|c|c|c|c|c|}
\hline \multirow[t]{2}{*}{ variable } & & Before & After Intervention & \multirow[t]{2}{*}{$P$ value } \\
\hline & & Mean $\pm S D$ & Mean $\pm S D$ & \\
\hline \multirow{2}{*}{$\begin{array}{l}\text { Physical activity level } \\
\text { (MET/Min) }\end{array}$} & Control & $4.17 \pm 2.09$ & $2.37 \pm 1.08$ & $<0.001$ \\
\hline & Intervention & $4.79 \pm 1.70$ & $5.48 \pm 1.92$ & $<0.001$ \\
\hline$P$ value & & 0.17 & $<0.001$ & \\
\hline \multirow[t]{2}{*}{ Weight (Kg) } & Control & $72.17 \pm 13.63$ & $79.00 \pm 15.31$ & $<0.001$ \\
\hline & Intervention & $72.41 \pm 13.25$ & $74.57 \pm 13.43$ & $<0.001$ \\
\hline$P$ value & & 0.94 & 0.20 & \\
\hline
\end{tabular}

Another comparison between the groups was based on the intensity of activities performed by participants in both groups. At the beginning of the study, there was no significant difference between the two groups based on the intensity of activity in the four categories of activity; sitting, light, moderate, and intense. For all activities in both groups, compared to the beginning, the intensity of activities at the end of the intervention showed a significant difference (intra-group comparison). Comparison of the two groups based on different categories of activity intensity at the end of the intervention showed a nonsignificant difference in sitting activity, while the intervention yielded significant differences between groups based on light, moderate, and severe activities (Table 3 ). So, the intervention can make a significant difference between groups in term of the intensity of activities.

Table 3-Comparison of mean and standard deviation of different levels of physical activity before and after the intervention among the groups 


\begin{tabular}{|c|c|c|c|c|}
\hline \multicolumn{2}{|l|}{ Variable } & $\begin{array}{l}\text { Control } \\
\text { Mean } \pm S D\end{array}$ & $\begin{array}{l}\text { Intervention } \\
\text { Mean } \pm S D\end{array}$ & P value \\
\hline \multirow[t]{2}{*}{ Sedentary } & Before & $124.49 \pm 103.39$ & $142.87 \pm 133.69$ & 0.52 \\
\hline & After & $26.55 \pm 14.14$ & $27.01 \pm 12.94$ & 0.89 \\
\hline$P$ value & & $<0.001$ & $<0.001$ & \\
\hline \multirow[t]{2}{*}{ Moderate } & Before & $174.72 \pm 224.28$ & $240.83 \pm 258.99$ & 0.25 \\
\hline & After & $11.33 \pm 18.06$ & $58.20 \pm 34.17$ & $<0.001$ \\
\hline$P$ value & & $<0.001$ & $<0.001$ & \\
\hline \multirow[t]{2}{*}{ Light } & Before & $352.36 \pm 291.33$ & $444.25 \pm 317.98$ & 0.21 \\
\hline & After & $37.50 \pm 22.20$ & $89.44 \pm 30.26$ & $<0.001$ \\
\hline$P$ value & & $<0.001$ & $<0.001$ & \\
\hline \multirow[t]{2}{*}{ Sever } & Before & $5.06 \pm 13.83$ & $11.31 \pm 22.19$ & 0.16 \\
\hline & After & $0.59 \pm 2.04$ & $1.66 \pm 2.50$ & 0.05 \\
\hline$P$ value & & 0.01 & 0.07 & \\
\hline
\end{tabular}

A comparison of changes in the type of activities (i.e., exercise, daily, and home activities) between the groups at the beginning and end of the intervention showed that the two groups did not differ significantly in any types of activities in the beginning phase. Meanwhile, at the end of intervention, the two groups showed significant differences in the three types of activities. Intra-group comparison based on activity types indicated a significant difference between the groups at the end of the study; however, the difference was more significant in the treatment group (Table 4).

Table 4 - Comparison of mean and standard deviation of physical activity type before and after intervention in the two groups 


\begin{tabular}{|lllll|}
\hline variable & & control & \multicolumn{1}{l}{ Intervention } & P value \\
\cline { 2 - 4 } & & Mean \pm SD & Mean \pm SD & \\
\hline Exercise & Before & $17.42 \pm 33.51$ & $36.44 \pm 51.71$ & 0.07 \\
\cline { 2 - 5 } & After & $1.77 \pm 3.49$ & $13.09 \pm 6.57$ & $<0.001$ \\
\hline P value & & 0.01 & 0.01 & \\
\hline Home activity & Before & $432.35 \pm 406.47$ & $537.99 \pm 424.88$ & 0.29 \\
\cline { 2 - 5 } & After & $38.19 \pm 26.47$ & $100.85 \pm 39.92$ & $<0.001$ \\
\hline P value & & $<0.001$ & $<0.001$ & \\
\hline Occupational & Before & $26.06 \pm 53.56$ & $44.21 \pm 87.16$ & 0.29 \\
\cline { 2 - 5 } & After & $4.34 \pm 10.27$ & $12.18 \pm 18.95$ & 0.03 \\
\hline P value & & 0.01 & 0.01 & \\
\hline
\end{tabular}

\section{Discussion}

The current study assessed the impact of virtual network-based training in mobile phones on the change in physical activity patterns of women during pregnancy. The study revealed the effectiveness of the designed training program on the change in women's physical activity patterns.

In the post-intervention phase, the mean level of physical activity in the intervention group was significantly different from the control group and its level in the start phase. The level of physical activity in the treatment group was increased at the end of intervention, while decrease in physical activity level was occurred in the control group. These findings are consistent with the results of Jane et al. (2018), who conducted an educational intervention to track the changes in physical activity behavior. At the end of intervention, they reported a significant reduction in the inactivity of the training group [23].

The results of the present study showed that although the mean intensity of physical activity decreased in both groups, the mean scores in the treatment group were significantly higher compared to the control group. This means that the reduction in the intensity of physical activity in the treatment group was much less than the control group. This may indicate that women had gained a better understanding about the importance of physical activity during pregnancy and therefore showed a greater tendency to do physical activity in the category of light and medium activity level, plus home activity, and exercise than the control group. Similar results have obtained in a web-based intervention study by Rothert et al. (2006) and by Wilkinson et al $(2016)[22,24]$. In the present research, the decrease in the intensity of physical activity in both groups could be due to the increase in pregnancy age. Moreover, pregnant women at this age of pregnancy face certain limitations due to fetal growth and weight gain during pregnancy. In line with the present project, on week 36 of pregnancy, Szmeja et al. (2014) has not found a statistically significant difference between the physical activity level of pregnant women in the control and treatment group, after 
distant training by educational DVDs during the third trimester [25]. Also, Cohen et al. (2013) obtained similar results in their study [26].

The findings of the present study are in line with those of Tehrani et al. (2016), who assessed the effect of a modern communication technology-based intervention (i.e., multi-media, internet, and mobile phones) on improving the women's physical activity [27]. They showed that the amount of awareness, attitude, and physical activity increased in the treatment group, and finally they concluded that training via mobile phones can have positive effects on the physical activity level of women.

Gaderpanah et al. (2017) concluded that training could lead to an increase in physical activity level or less reduction of the intensity of physical activity. In their study, the mean physical activity intensity increased considerably in the treatment group [28]. Moreover, after the educational intervention, an increase in the intensity of activities was seen. In the present study, the technology-based educational intervention led to the prevention of a sharp decrease in the intensity of physical activity in the intervention group compared to the control group. This difference in the effect of the intervention may be due to the difference in the intervention type. More ever, in their study, the training intervention was performed in the form of counseling method based on $5 \mathrm{~A}$ Model.

According to the results of the present research, the virtual network-based intervention in the mobile platform can make a significant difference between the two groups in all three categories of activity intensity (i.e., light, moderate, intense), but did not make a significant difference in sitting activity ( $p=$ 0.89). These findings are consistent with those of Bisson et al. (2015), as in their study technology-based education led to an increase in all three categories of activity including light, moderate, and intense activities [29]. Findings of the present research like many other the previous studies indicated that the mean exercise activity of women is generally low. For instance, in a study from Thailand, most women considered exercise as an effective and advantageous activity during pregnancy and believed that its benefits outweighed its disadvantages; nevertheless, they had little physical activity during pregnancy. The results of their study showed that $40 \%$ of pregnant women, without any medical limitations, passed this period without exercising [30].

Some studies suggest that mean of the total activities has not been statistically different in pregnancy [8, $25,31]$, which examined the effect of educational intervention on women's physical activity levels (i.e., light, moderate, intense, and sedentary), showed that the use of mobile software to intervene in the field of physical activity during pregnancy does not cause a significant change in the moderate and severe levels of activity in intervention group [32]. These findings are in contrast with our results. The difference in the results can partially be attributed to differences in the target population and differences in the software used for the intervention, how to implement and follow up the intervention, the duration of the intervention, and the lack of active training in the intervention. Another inconsistent study is the one conducted by Magann et al. (2002). Such discrepancies might be due to differences in educational content, how the intervention is implemented, cultural differences, and the resulting changes [33]. 
Important limitations of the present study include the vulnerability of the pregnancy, the lack of access to a smartphone for some people, and the fear of complications in some people during pregnancy. In this study, the possibility of drop out in the sample size was controlled effectively. Despite the positive effect of intervention via the social network in the mobile context on the behavior of women's physical activity, it was not possible to control and obligate them to perform all physical activities quietly, and also the respect to their freedom to some extent affected the results. However, the reminders through the posted messages in the created social group, phone calls, and frequent follow-ups with short intervals could somehow reduce these effects. Other limitations were the low speed of the internet and in some cases the internet interruption or problems related to filtering of used social network (Telegram) in Iran.

Nevertheless, it seems that interventions based on social networks in the mobile context can have a positive effect on the level of physical activity during pregnancy.

One of the strengths of the present study is the frequent and short-interval follow-up and the regular repetition of the educational message in the virtual group. Another advantage of our study was recruiting a group of relevant specialists (including a nutritionist, physical activity specialist, health specialist, and midwife) for preparing the useful training program for each area. Moreover, the use of alternative social networking to ensure timely access to educational content and programs was another advantage of the present study.

\section{Conclusion}

Educational intervention based on the social networks in the context of mobile phones has an effective role in changing the behavior of women during pregnancy and can to motivate them and change their behavior for promoting their health during the pregnancy. Therefore it can be recommended to provide the educational and interventional programs during pregnancy, especially based on the eHealth and mHealth in changing women's behavior in physical activity during this life period.

\section{Abbreviations}

PPAQ: Demographic and Pregnancy Physical Activity Questionnaire, BMI: Body Mass Index, MET: Metabolic Equivalents, UMSU: Urmia Medical Sciences University

\section{Declarations}

\section{Acknowledgments}

We would like to thank the UMSU Vice-Chancellor of Research and Technology, the health center staff of Urmia, and pregnant mothers who accompanied the research team in all stages of the study.

\section{Authors' contributions}


ET, HM contributed to the design of the manuscript. JR and DV contributed to the implementation and analysis plan. ET contributed to data collection. ET, HM and DV have written the first draft of this manuscript. All authors read and approved the final manuscript.

Funding: This study was supported by UMSU financially.

\section{Availability of data and materials}

The dataset used in this study is available from the corresponding author on reasonable request.

\section{Ethics approval and consent to participate}

The ethics committee of Urmia University of Medical Sciences approved the study (Ethical code: IR.UMSU.REC.1397.162). This study was registered in the Iranian Registry of Clinical Trials (Registration code: IRCT20151004024340N15, Date: 2020/06/05). The participants were fully informed about the study goals. Each participant provided a written consent form prior to the participation. All study protocols including interventions, procedures and methods was according to the principles of Helsinki declaration. Participants were allowed to quit the study whenever desired.

\section{Consent for publication}

Not applicable

\section{Competing Interest}

The authors declare no conflict of interest.

\section{References}

1. Siega-Riz AM, Evenson KR, Dole N: Pregnancy-related weight gain-a link to obesity? Nutrition reviews 2004, 62(suppl_2):S105-S111.

2. Garber CE, Blissmer B, Deschenes MR, Franklin BA, Lamonte MJ, Lee I-M, Nieman DC, Swain DP: Quantity and quality of exercise for developing and maintaining cardiorespiratory, musculoskeletal, and neuromotor fitness in apparently healthy adults: guidance for prescribing exercise. 2011.

3. Holmes $\mathrm{V}$, Wallace J: Haemostasis in normal pregnancy: a balancing act? Biochemical society transactions 2005, 33(2):428-432.

4. Hebden L, Cook A, Van der Ploeg H, King L, Bauman A, Allman-Farinelli MJJoHN, Dietetics: A mobile health intervention for weight management among young adults: a pilot randomised controlled trial. 2014, 27(4):322-332.

5. Organization WH: Global status report on noncommunicable diseases 2014: World Health Organization; 2014. 
6. Duncombe D, Wertheim EH, Skouteris H, Paxton SJ, Kelly L: Factors related to exercise over the course of pregnancy including women's beliefs about the safety of exercise during pregnancy. Midwifery 2009, 25(4):430-438.

7. Teixeira PJ, Carraça EV, Marques MM, Rutter H, Oppert J-M, De Bourdeaudhuij I, Lakerveld J, Brug J: Successful behavior change in obesity interventions in adults: a systematic review of self-regulation mediators. BMC medicine 2015, 13(1):1-16.

8. Guelinckx I, Devlieger R, Mullie P, Vansant G: Effect of lifestyle intervention on dietary habits, physical activity, and gestational weight gain in obese pregnant women: a randomized controlled trial. The American journal of clinical nutrition 2010, 91(2):373-380.

9. Stafne S, Salvesen K, Romundstad P, Stuge B, Mørkved S: Does Regular Exercise During Pregnancy Influence Lumbopelvic Pain? A Randomized Control Trial. Obstetric Anesthesia Digest 2013, 33(2):89-90.

10. Downs DS, Hausenblas HA: Women's exercise beliefs and behaviors during their pregnancy and postpartum. Journal of midwifery \& women's health 2004, 49(2):138-144.

11. Hassall J: Exercise in pregnancy: A review of current evidence and guidelines. Essentially Midirs 2011, 2(1):39-42.

12. Salmon P: Effects of physical exercise on anxiety and sensitivity to stress. London \& New York: Rutledge. In.: Taylor \& Francis Group; 2008.

13. Kader M, Naim-Shuchana S: Physical activity and exercise during pregnancy. The European Journal of Physiotherapy 2014, 16(1):2-9.

14. Evenson KR, Wen F: National trends in self-reported physical activity and sedentary behaviors among pregnant women: NHANES 1999-2006. Preventive medicine 2010, 50(3):123-128.

15. Schmidt MD, Pekow P, Freedson PS, Markenson G, Chasan-Taber L: Physical activity patterns during pregnancy in a diverse population of women. Journal of Women's Health 2006, 15(8):909-918.

16. Fell DB, Joseph K, Armson BA, Dodds L: The impact of pregnancy on physical activity level. Maternal and child health journal 2009, 13(5):597.

17. Gregory KD, Niebyl JR, Johnson TR: Preconception and prenatal care: part of the continuum. GABBE, SG et al Obstetrics: normal and problem pregnancies 2016, 7:101-124.

18. Lloyd A: Social media, help or hindrance: What role does social media play in young people's mental health. Psychiatr Danub 2014, 26(Suppl 1):340-346.

19. Nour M, Chen J, Allman-Farinelli M: Efficacy and external validity of electronic and mobile phonebased interventions promoting vegetable intake in young adults: systematic review and metaanalysis. Journal of medical Internet research 2016, 18(4):e5082.

20. Hurt K, Walker RJ, Campbell JA, Egede LE: mHealth interventions in low and middle-income countries: a systematic review. Global journal of health science 2016, 8(9):183.

21. King AC, Ahn DK, Oliveira BM, Atienza AA, Castro CM, Gardner CD: Promoting physical activity through hand-held computer technology. American journal of preventive medicine 2008, 34(2):138- 
142.

22. Rothert K, Strecher VJ, Doyle LA, Caplan WM, Joyce JS, Jimison HB, Karm LM, Mims AD, Roth MA: Web-based weight management programs in an integrated health care setting: a randomized, controlled trial. Obesity 2006, 14(2):266-272.

23. Shigekawa E, Fix M, Corbett G, Roby DH, Coffman JJHA: The current state of telehealth evidence: a rapid review. 2018, 37(12):1975-1982.

24. Wilkinson A, Forbes A, Bloomfield J, Gee CF: An exploration of four web-based open and flexible learning modules in post-registration nurse education. International journal of nursing studies 2004 , 41(4):411-424.

25. Szmeja MA, Cramp C, Grivell RM, Deussen AR, Yelland LN, Dodd JM: Use of a DVD to provide dietary and lifestyle information to pregnant women who are overweight or obese: a nested randomised trial. BMC pregnancy and childbirth 2014, 14(1):1-11.

26. Cohen TR, Koski KG: Limiting excess weight gain in healthy pregnant women: importance of energy intakes, physical activity, and adherence to gestational weight gain guidelines. Journal of pregnancy 2013, 2013.

27. Tehrani $\mathrm{H}$, Kabootarkhani MH, Peyman N, Vahedian-Shahroodi M: The impact of new communications technology on promoting women's physical activity. Payesh (Health Monitor) 2016, 15(3):293-300.

28. Ghaderpanah N, Mohaddesi $\mathrm{H}$, Vahabzadeh D, Khalkhali $\mathrm{H}$ : The effect of $5 \mathrm{~A}$ model on behavior change of physical activity in overweight pregnant women. The Iranian Journal of Obstetrics, Gynecology and Infertility 2017, 20(9):101-114.

29. Bisson M, Alméras N, Dufresne SS, Robitaille J, Rhéaume C, Bujold E, Frenette J, Tremblay A, Marc I: A 12-week exercise program for pregnant women with obesity to improve physical activity levels: an open randomised preliminary study. PLoS One 2015, 10(9):e0137742.

30. Liamputtong P, Yimyam S, Parisunyakul S, Baosoung C, Sansiriphun N: Traditional beliefs about pregnancy and child birth among women from Chiang Mai, Northern Thailand. Midwifery 2005, 21(2):139-153.

31. Mummah SA, King AC, Gardner CD, Sutton SJIJoBN, Activity P: Iterative development of Vegethon: a theory-based mobile app intervention to increase vegetable consumption. 2016, 13(1):90.

32. Choi J, hyeon Lee J, Vittinghoff E, Fukuoka Y: mHealth physical activity intervention: a randomized pilot study in physically inactive pregnant women. Maternal and child health journal 2016, 20(5):1091-1101.

33. Magann EF, Evans SF, Weitz B, Newnham J: Antepartum, intrapartum, and neonatal significance of exercise on healthy low-risk pregnant working women. Obstetrics \& Gynecology 2002, 99(3):466472.

\section{Figures}




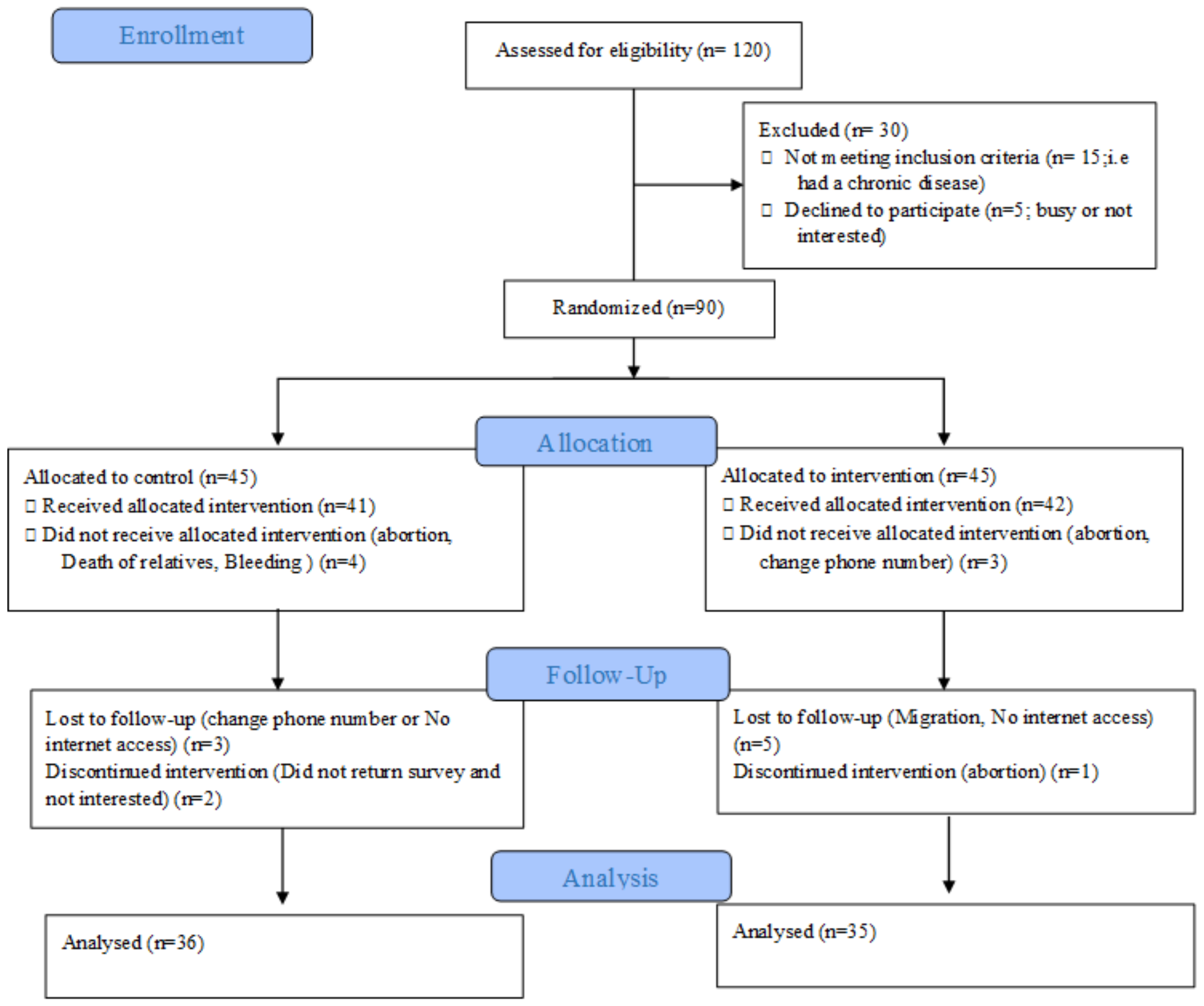

Figure 1

CONSORT Flowchart 